\title{
Analysis of the impact of ERK5, JNK, and P38 kinase cascades on each other: A systems approach
}

\author{
Pandurangan Sundaramurthy ${ }^{1}$, Sunita Gakkhar ${ }^{2}$ and Ramanathan Sowdhamini ${ }^{3,}$ * \\ ${ }^{1,2}$ Department of Mathematics, Indian Institute of Technology Roorkee, Roorkee - 247667, Uttarakhand, India; $;{ }^{1,3}$ National Center for Biological \\ Sciences, Tata Institute of Fundamental Research, UAS-GKVK campus, Bellary Road, Bangalore - 560 065, India; \\ R. Sowdhamini* - Email: mini@ncbs.res.in; * Corresponding author
}

received November 04, 2008; accepted November 11, 2008; published January 12, 2009

\begin{abstract}
:
The classical concept of linear pathways is being increasingly challenged by network representations, which emphasize the importance of interactions between components of a biological system, and motivates for adopting a system-level approach in biology. We have developed a dynamical system that integrates quantitative, dynamic and topological representation of network of ERK5 (Extracellular signal-regulated kinases 5), JNK(c-Jun N-terminal kinases) and P38 kinase cascades. We have observered that, the transient activation of ERK5, JNK1 and P38 $\beta$ kinase, and the persistent activation of JNK2, JNK3 and P38 $\delta$ kinase does not get affected due to the cross-talks between ERK5, JNK and P38 kinase cascades. But it is due to the cross - talks, the transiently activated P38 $\alpha$ kinase become inactivated, and the transiently activated P38 $\gamma$ kinase become persistently activated. The impacts of one-way cross-talks between the cascades are insignificant and differ from the impact of two-way cross-talks. We generate a hypothesis that, signaling pathways should be studied as a system by considering the cross-talks between the two adjacent cascades.
\end{abstract}

Keywords: cross-talks; ERK5, JNK and P38 kinase cascades; systems approach

\section{Background:}

Cells need to adjust to changes in the external environment, to respond to signals from neighboring cells. Binding of extracellular signaling molecules to cell-surface receptors such as G protein-coupled receptors (GPCRs) and receptor tyrosine kinases (RTKs) activates limited number of signaling pathways. These pathways do not simply transmit, but they also process, encode and integrate internal and external signals. MAPK (mitogen-activated protein kinase) signal transduction pathways regulate diverse cellular processes ranging from proliferation and differentiation to

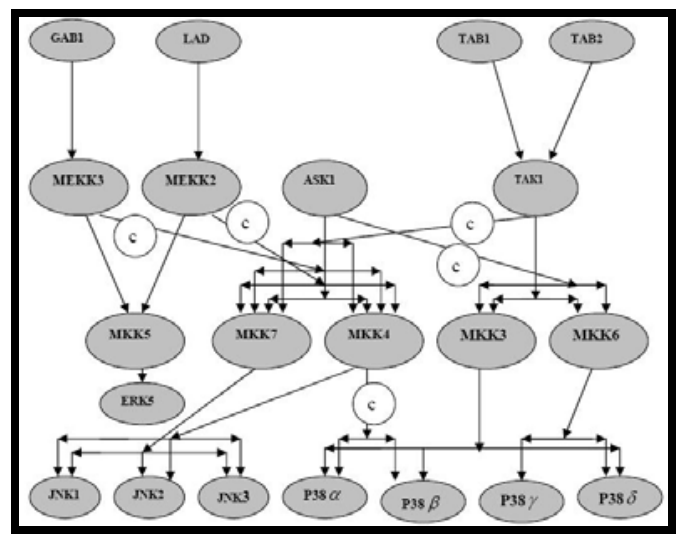

Figure 1: ERK5, JNK and P38 kinase cascades with cross-talks. C $=$ cross talk

ISSN 0973-2063 (online) 0973-8894 (print)

Bioinformation 3(6): 244-249 (2009) apoptosis [1]. Five families of MAPKs have been defined in mammalian cells: extra cellular signal-regulated kinases (ERK1 and ERK2); c-Jun N-terminal kinases (JNK1, JNK2 and JNK3); P38 kinase isozymes (P38 $\alpha, \mathrm{P} 38 \beta, \mathrm{P} 38 \gamma$ and P38ס); ERK3/ERK4; and ERK5. By and large, activation of ERKs has been linked to cell survival, whereas JNKs and P38 kinases are linked to induction of apoptosis. This dichotomy, however, is an oversimplification, and the actual roles of each MAPK cascade are highly cell-type specific and context- dependent [2]. 


\section{www.bioinformation.net}

\section{Hypothesis}

In the processes of cellular signaling, protein-protein interactions play a central role. Protein kinases are enzymes that covalently attach phosphate to the side chain of serine, threonine, or tyrosine of specific proteins inside cells and protein phosphatases remove the phosphates that were transferred to the protein substrate by the kinase. In this manner, the action of MAPKs and protein phosphatases reciprocally and rapidly alter the behavior of cells as they respond to changes in their environment [2]. A MAPKKK that is activated by extracellular stimuli phosphorylates a MAPKK on its serine and threonine residues. This MAPKK activates a MAPK through phosphorylation of its serine and tyrosine residues. The activated MAPKs, in turn, phosphorylate specific serines and threonines of target protein substrates and regulate cellular activities ranging from gene expression, mitosis, movement, metabolism, and programmed death [2]. Based on the information's given in JNK and P38 pathway diagrams deposited in the Science's STKE (Signal Transduction Knowledge Environment) database [3, 4], we have drawn the diagram (Figure 1) to depict ERK5, JNK and P38 Kinase cascades with cross-talks.
ERK5 is a MAP Kinase regulated by a wide range of mitogens and cellular stresses. The potentially crucial role of ERK5 in cancers and heart diseases make this cascade highly attractive for the development of new therapeutic strategies to treat pathological conditions that are resistant to current therapies [5]. The JNKs consist of three isoforms: JNK1 and JNK2 are the products of alternative splicing of a single gene and are expressed in many tissues, but JNK3 is specifically expressed in neuronal tissue brain. Members of the JNK family play crucial roles in regulating responses to environmental stress, radiation, and growth factors, and in neural development, inflammation, and apoptosis [6]. Four isoforms of P38 MAP kinase: P38 $\alpha$, P38 $\beta$, P38 $\gamma$ and P38 $\delta$ have been identified. The P38 MAPKs play an important role in asthma and autoimmunity in humans and are activated by numerous physical and chemical stresses, including hormones, UV irradiation, ischemia, cytokines including interleukin-1 and tumor necrosis factor, osmotic shock and heat shock [7].

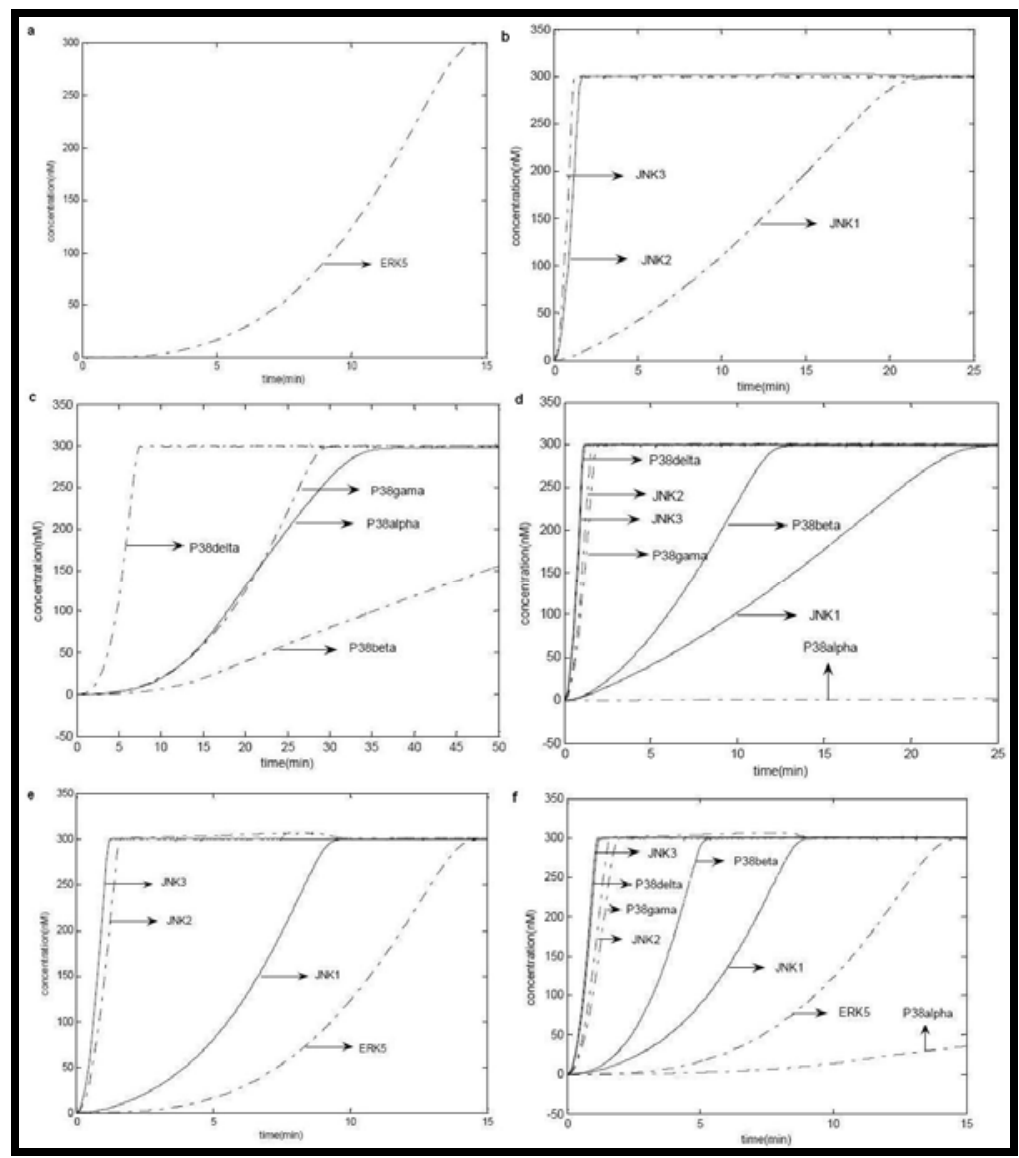

Figure 2: Results of simulation is given for (a) ERK5 cascade with unit-step input signals; (b) JNK cascade; (c) P38 kinase cascade with unit-step input signal; (d) JNK and P38 kinase cascades with cross-talks and unit-step input signal; (e) ERK5 and JNK cascades with cross-talks and unit-step input signals; (f) ERK5, JNK and P38 kinase cascades with cross-talks and unit-step input signals. 
Bioinformation

www.bioinformation.net

open access

\section{Hypothesis}

\begin{abstract}
Methodology:
See supplementary material for methodology and mathematical formulation.

\section{Discussion:}

We have assist-MATLAB to numerically integrate (simulation) the system of differential equations using Adams-Bash forth algorithm and considered the plots, which represents the activation of ERK5, JNKs and P38 kinases only for analysis. We have observed that, the qualitative behavior of the stimulus / response curves does not change for a range of input signals $(1-5 \mathrm{nM})$ and for a range of reaction rate constants $(0.001-3$ $\mathrm{nM} / \mathrm{s}$ ). See Figure 2 for simulation results. It might be due to the robust nature of the kinase cascades. Also, we have taken the molar concentrations for MAPKs, MAPKKs and MAPKKKs such that molar concentration of MAPKs are lesser than that of MAPKKs and MAPKKKs, and the molar concentration of MAPKKs and MAPKKKs are equal as it has been observed and used in practice. We have taken the values of Michaelis-Menten's constants such that, the ratio of the molar concentration of enzymes and the Michaelis-Menten's constants are in the range of $10-20$.
\end{abstract}

We have observed that, the transient activation of ERK5, JNK1 and P38 $\beta$ kinase, and the persistent activation of JNK2, JNK3 and P38 $\delta$ kinase does not get affected due to the cross talks between ERK5, JNK and P38 kinase cascades. But it is due to the cross - talks, the transiently activated P38 $\alpha$ kinase become inactivated, and the transiently activated P38 $\gamma$ kinase become persistently activated. Also, the influence of ERK5 cascade on JNK and P38 kinase cascades is insignificant, might be due to the one way nature of the cross -talks between ERK5 and JNK cascades. Transient activation of ERK5 may trigger cell proliferation [11], persistent activation of JNK2 and JNK3 is associated with apoptosis and the transient activation of JNK1 is associated with cell survival [12, 13]. The persistent activation of P38 $\gamma$ kinases might mediate process like mitogenesis, cell fate induction [14], and [16] may result in an anti-angiogenic phenotype that contributes to endothelial dysfunction [15].

\section{Conclusion:}

The impacts of one-way cross-talks between the cascades are insignificant and differ from the impact of two-way cross-talks. We generate a hypothesis that, signaling pathways should be studied as a system by considering the cross - talks between the two adjacent cascades.

\section{References:}

[01] L. Chang and M. Karin, Nature, 410: 37 (2001) [PMID: 11242034]

[02] G. L. Johnson and R. Lapadat, Science, 298: 1911(2002) [PMID: 12471242]

[03] http://stke.sciencemag.org/cgi/cm/stkecm;CMP_10827

[04] http://stke.sciencemag.org/cgi/cm/stkecm;CMP_10958

[05] Y. Kato et al., EMBO J., 16: 7054 (1997) [PMID: 9384584]

[06] R. J. Davis, Cell, 103: 239 (2000) [PMID: 11057897]

[07] J. Han et al., Nature, 20: 296 (1997) [PMID: 9069290]

[08] O. Wolkenhauer et al., Biochem SocTrans., 33: 507 (2005) [PMID: 15916553]

[09] R. Heinrich et al., Mol Cel., 9: 957 (2002) [PMID: 12049733]

[10] B. N. Kholodenko et al., Proc Natl Acad Sci U S A., 99: 12841 (2002) [PMID: 12242336]

[11] L. Scapoli et al., Oncogene, 23: 805 (2004) [PMID: 14737115]

[12] E. Shaulian et al., Cell, 103: 6 (2000) [PMID: 11136975]

[13] C. Reinhard et al., EMBO J., 16: 5 (1997) [PMID: 9118946]

[14] J. E. Ferrell, Trends Biochem Sci., 21: 12 (1996) [PMID: 9009826]

[15] M. E. McMullen et al., J Biol Chem., 280: 22 (2005) [PMID: 15790570]

Edited by $P$. Kangueane

Citation: Sundaramurthy et al., Bioinformation 3(6): 244-249 (2009)

License statement: This is an open-access article, which permits unrestricted use, distribution, and reproduction in any medium, for non-commercial purposes, provided the original author and source are credited.

\section{Supplementary material \\ Methodology:}

Mathematical model:

The temporal dynamics of signaling cascades is described by ordinary differential equations, which are known as chemicalkinetics equations. The derivation begins by listing all chemical transformations (Figure S1), thereby providing a kinetic scheme of a kinase cascade. A basic assumption of this approach is that the cell presents a well-stirred biochemical reactor. The following simple model of an enzyme kinetic reaction serves as a subsystem for signaling cascade [8].

$\mathrm{S}+\mathrm{E} \underset{\mathrm{k} \mathbf{2}}{\stackrel{\mathrm{kl}}{\longrightarrow}} \mathrm{SE} \stackrel{\mathrm{k3}}{\longrightarrow} \mathrm{P}+\mathrm{E}$

E denotes the enzyme, SE is the substrate - enzyme complex and P is the product. Referring to the law of mass action, one arrives at the state - space model 


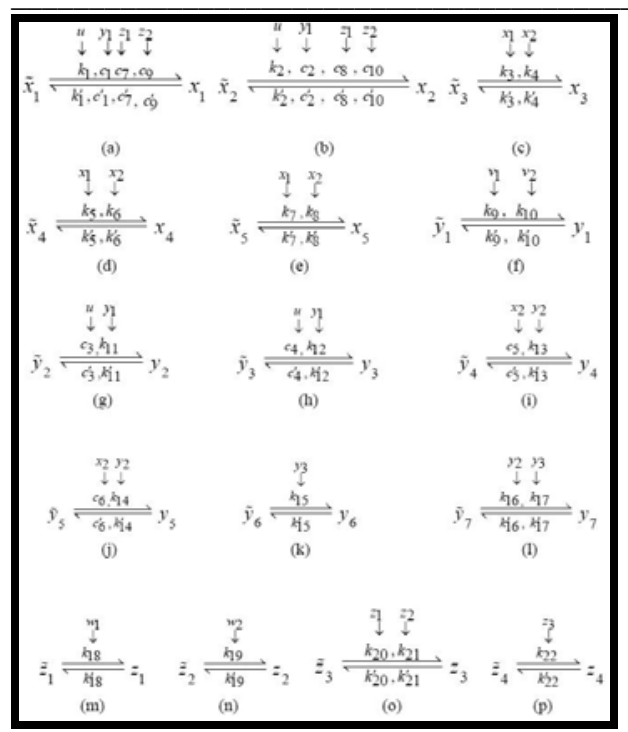

Figure S1: (a) to (p): Reaction schemes of activation (phosphorylation) and inactivation (de-phosphorylation) of all the proteins in ERK, JNK and P38 kinase cascades with cross-talks.

$$
\left.\begin{array}{l}
\frac{d}{d t} E(t)=-k_{1} E(t) S(t)+k_{2} S(t) E(t)+k_{3} S(t) E(t) \\
\frac{d}{d t} S(t)=-k_{1} E(t) S(t)+k_{2} S(t) E(t) \\
\frac{d}{d t} S(t) E(t)=k_{1} E(t) S(t)-k_{2} S(t) E(t)-k_{3} S(t) E(t) \\
\frac{d}{d t} P(t)=k_{3} S(t) E(t) .
\end{array}\right\}
$$

Where $k_{1}, k_{2}$ and $k_{3}$ are reaction rate constants.

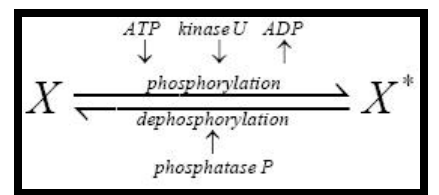

Figure S2: Biochemical reaction

Modeling system of ERK5, JNK and P38 kinase cascades with cross-talks

In signaling, activation/inactivation of proteins corresponds to phosphorylation/ dephosphorylation. Denoting the kinase as U, the phosphatase as $\mathrm{P}$ and the protein as $\mathrm{X}$, and assuming a constant phosphatase, (Figure S2) illustrates a simple biochemical model of a signaling step is given by $\dot{x}=v_{1}(u, \tilde{x})-v_{2}(x)$, where $v_{1}($.$) and v_{2}($.$) are mappings, describing the reaction rates for$ phophorylation and dephophorylation respectively [8]. We write $\tilde{X}$ for the non - phosphorylated form of the protein $\mathrm{X}$, $\mathrm{u}$ for the kinase $\mathrm{U}$, and $\mathrm{x}$ corresponds to the activated protein $X^{*}$. Referring to a power-law representation, one would have $v_{1}(u, \tilde{x})=k_{1} u^{a} \tilde{x}^{b}, v_{2}(x)=k_{2} x^{c}$ where we choose $a=b=c=1$, such that $\dot{x}=k_{1} u(t) \tilde{x}(t)-k_{2} x(t)$. If we assume that the total $\bar{x}=\tilde{x}(t)+x(t)$ is constant for all time $t$, we require only this one differential 
equation $\dot{x}=k_{1} u(t)(\bar{x}(t)-x(t))-k_{2} x(t)$ to model a signaling step [9]. In our model we have assumed MichaelisMenten kinetics [10].

$\dot{x}=\underbrace{\frac{k_{1} u(t)(\bar{x}(t)-x(t))}{K m 1+(\bar{x}(t)-x(t))}}_{\text {phosphorylation }}-\underbrace{\frac{k_{2} x(t)}{K m 2+x(t)}}_{\text {dephosphorylation }}$

To model the entire ERK5, JNK and P38 Kinase cascades with cross-talks (Figure 1), we have the following state variables representing the concentration of each protein involved in the system (Figure 1). Let $u=$ ASK1, $v_{1}=$ TAB1 (TAK1 (Transforming growth factor-beta-activated kinase 1)binding protein 1), $v_{2}=$ TAB2 (TAK1 binding protein 2), $w_{1}=\mathrm{GAB} 1(\mathrm{Grb} 2$-associated binder 1$), w_{2}=\mathrm{LAD}($ Lck-associated adapter $), x_{1}(t)=\mathrm{MKK} 7, \quad x_{2}(t)=\mathrm{MKK} 4, \quad x_{3}(t)=\mathrm{JNK} 1$, $x_{4}(t)=\mathrm{JNK} 2, \quad x_{5}(t)=\mathrm{JNK} 3, \quad y_{1}(t)=\mathrm{TAK} 1, \quad y_{2}(t)=\mathrm{MKK} 3, y_{3}(\mathrm{t})=\mathrm{MKK} 6, y_{4}(t)=\mathrm{P} 38 \alpha, \quad y_{5}(t)=\mathrm{P} 38 \beta, \quad y_{6}(t)=$ P38 $\gamma, y_{7}(t)=\mathrm{P} 38 \delta, z_{1}(t)=\mathrm{MEKK} 3, z_{2}(t)=\mathrm{MEKK} 2, z_{3}(t)=\mathrm{MKK} 5$, and $z_{4}(t)=\mathrm{ERK} 5$. Also, let $\left\{x_{i}(t)\right\}_{i=1 \text { to } 5}$, $\left\{y_{j}(t)\right\}_{j=1 \text { to } 7}$ and $\left\{z_{k}(t)\right\}_{k=1 \text { to } 4}$ are the activated (phosphorylated) form of the proteins and the tilted ones are corresponds to the inactivated (non-phosphorylated) in JNK and P38 and ERK5 Kinase cascades respectively. We assume that the total concentrations $\left\{\bar{x}_{i}=\tilde{x}_{i}(t)+x_{i}(t)\right\}_{i=1 \text { to } 5}\left\{\bar{y}_{j}=\tilde{y}_{j}(t)+y_{j}(t)\right\}_{j=1 \text { to } 7}$ and $\left\{\bar{z}_{k}=\tilde{z}_{k}(t)+z_{k}(t)\right\}_{k=1 \text { to } 4}$ are constant for all t.

Based on the reaction schemes described in (Figure 3(a) - 3(p)), a set of differential equations (3a)-(3p) have been developed to form the dynamic system, to analyse the impact of cross-talks between the ERK5, JNK and P38 Kinase cascades (Figure 1).

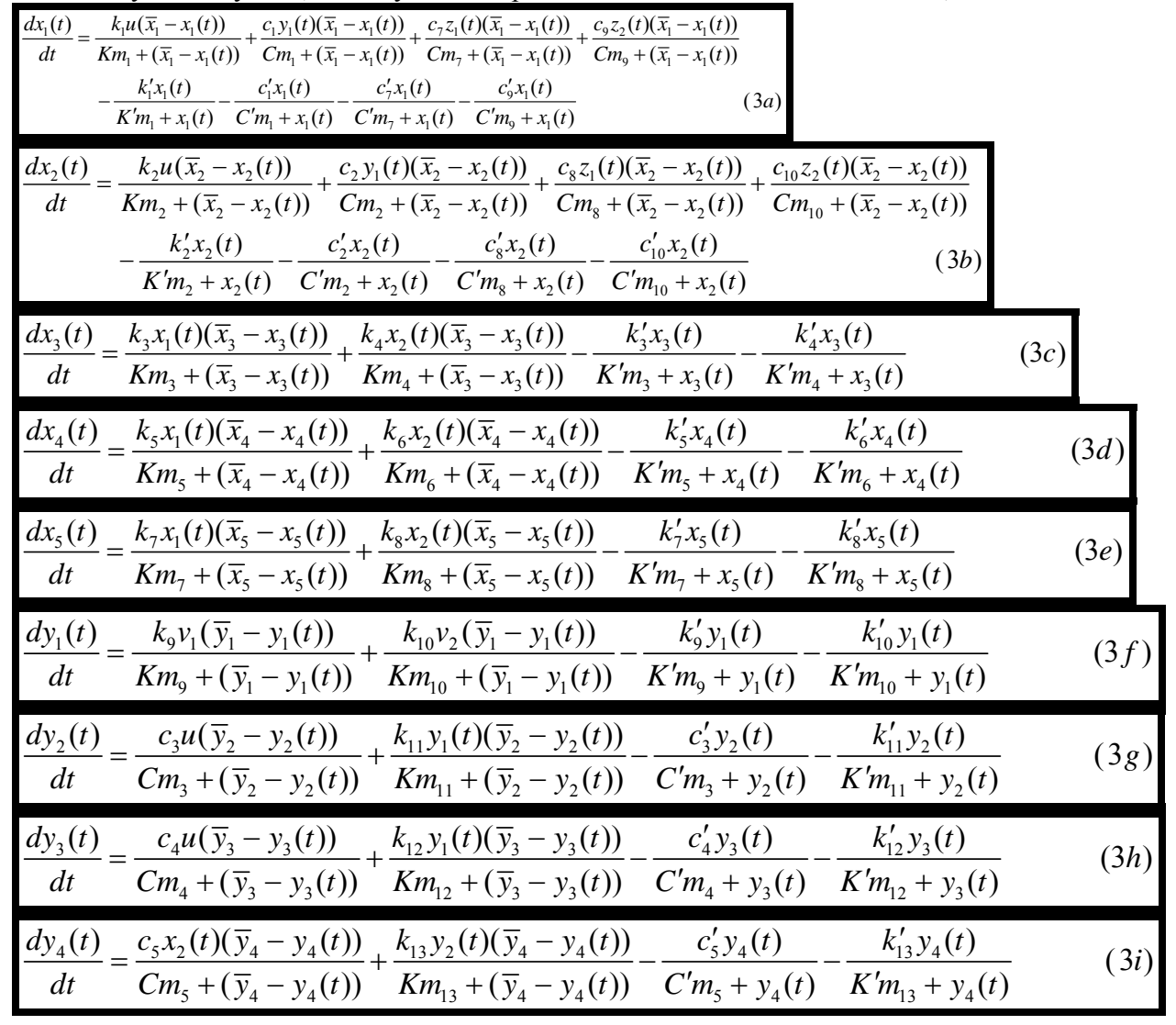




\section{www.bioinformation.net}

\section{Hypothesis}

\begin{tabular}{l}
\hline$\frac{d y_{5}(t)}{d t}=\frac{c_{6} x_{2}(t)\left(\bar{y}_{5}-y_{5}(t)\right)}{C m_{6}+\left(\bar{y}_{5}-y_{5}(t)\right)}+\frac{k_{14} y_{2}(t)\left(\bar{y}_{5}-y_{5}(t)\right)}{K m_{14}+\left(\bar{y}_{5}-y_{5}(t)\right)}-\frac{c_{6}^{\prime} y_{5}(t)}{C^{\prime} m_{6}+y_{5}(t)}-\frac{k_{14}^{\prime} y_{5}(t)}{K^{\prime} m_{14}+y_{5}(t)}$ \\
$\frac{d y_{6}(t)}{d t}=\frac{k_{15} y_{3}(t)\left(\bar{y}_{6}-y_{6}(t)\right)}{K m_{15}+\left(\bar{y}_{6}-y_{6}(t)\right)}-\frac{k_{15}^{\prime} y_{6}(t)}{K^{\prime} m_{15}+y_{6}(t)}$ \\
$\frac{d y_{7}(t)}{d t}=\frac{k_{16} y_{2}(t)\left(\bar{y}_{7}-y_{7}(t)\right)}{K m_{16}+\left(\bar{y}_{7}-y_{7}(t)\right)}+\frac{k_{17} y_{3}(t)\left(\bar{y}_{7}-y_{7}(t)\right)}{K m_{17}+\left(\bar{y}_{7}-y_{7}(t)\right)}-\frac{k_{16}^{\prime} y_{7}(t)}{K^{\prime} m_{16}+y_{7}(t)}-\frac{k_{17}^{\prime} y_{7}(t)}{K^{\prime} m_{17}+y_{7}(t)}$ \\
$\frac{d z_{1}(t)}{d t}=\frac{k_{18} w_{1}\left(\bar{z}_{1}-z_{1}(t)\right)}{K m_{18}+\left(\bar{z}_{1}-z_{1}(t)\right)}-\frac{k_{18}^{\prime} z_{1}(t)}{K^{\prime} m_{18}+z_{1}(t)}$ \\
$\frac{d z_{2}(t)}{d t}=\frac{k_{19} w_{2}\left(\bar{z}_{2}-z_{2}(t)\right)}{K m_{19}+\left(\bar{z}_{2}-z_{2}(t)\right)}-\frac{k_{19}^{\prime} z_{2}(t)}{K^{\prime} m_{19}+z_{2}(t)}$ \\
$\frac{d z_{3}(t)}{d t}=\frac{k_{20} z_{1}(t)\left(\bar{z}_{3}-z_{3}(t)\right)}{K m_{20}+\left(\bar{z}_{3}-z_{3}(t)\right)}+\frac{k_{21} z_{2}(t)\left(\bar{z}_{3}-z_{3}(t)\right)}{K m_{21}+\left(\bar{z}_{3}-z_{3}(t)\right)}-\frac{k_{20}^{\prime} z_{3}(t)}{K^{\prime} m_{20}+z_{3}(t)}-\frac{k_{21}^{\prime} z_{3}(t)}{K^{\prime} m_{21}+z_{3}(t)}$ \\
$\frac{d z_{4}(t)}{d t}=\frac{k_{22} z_{3}(t)\left(\bar{z}_{4}-z_{4}(t)\right)}{K m_{22}+\left(\bar{z}_{4}-z_{4}(t)\right)}-\frac{k_{22}^{\prime} z_{4}(t)}{K^{\prime} m_{22}+z_{4}(t)}$ \\
\hline$K_{i}^{\prime} i$
\end{tabular}

Where $k_{i}, k_{i}^{\prime} ; i=1$ to22 and $c_{j}, c_{j}^{\prime} ; j=1$ to10 are reaction rate constants and

$K m_{i}, K^{\prime} m_{i} ; i=1$ to 22 and $\mathrm{Cm}_{j}, C^{\prime} m_{j} ; j=1$ to10 are Michaelis-Menten's constants. The parameter values are given in (Table 1 under supplementary material).

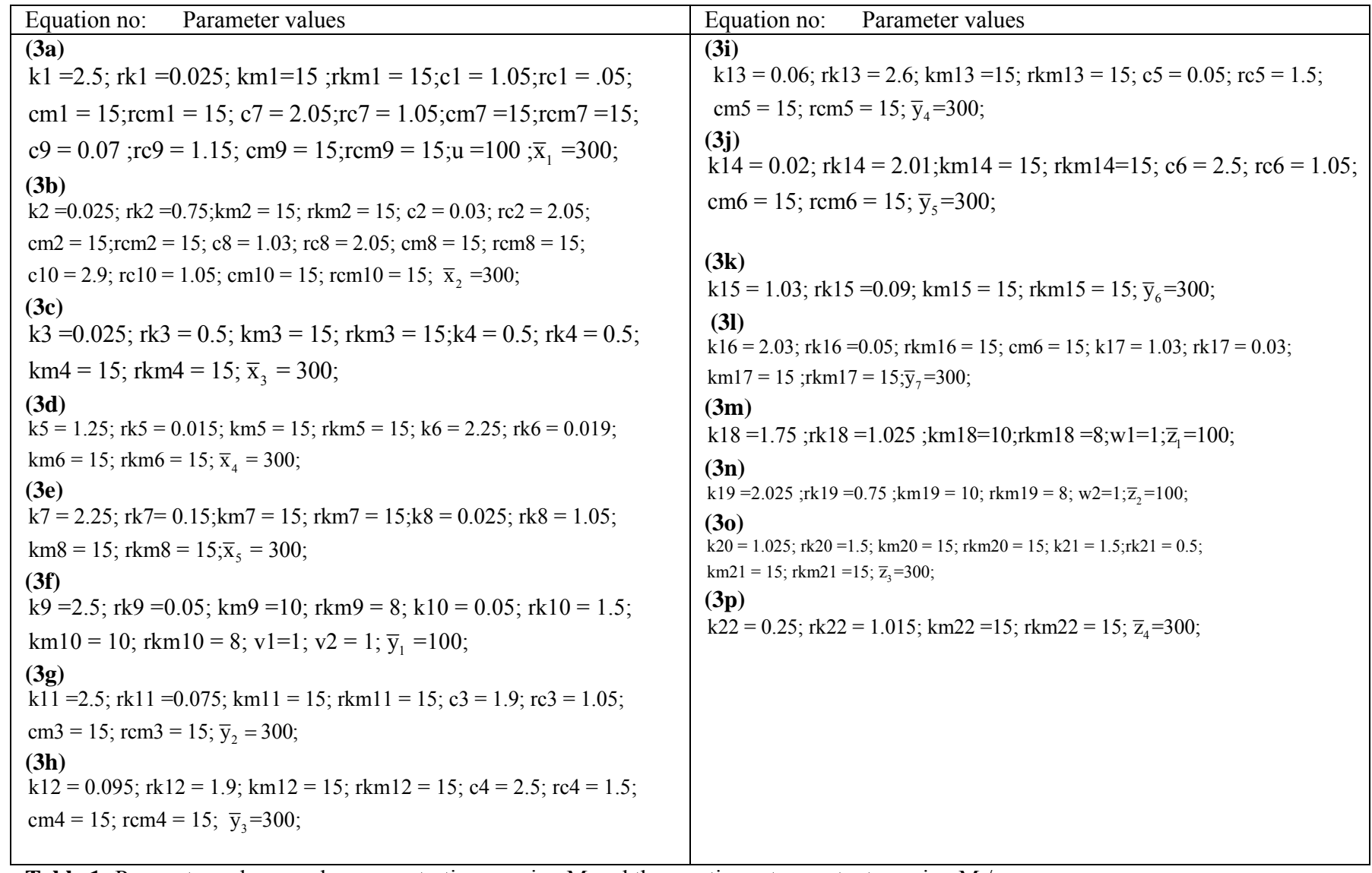

Table 1: Parameter values, molar concentrations are in $\mathrm{nM}$ and the reaction rate constants are in $\mathrm{nM} / \mathrm{s}$. 